\title{
Tumor necrosis factor- $\alpha$ antibodies (infliximab, adalimumab and certolizumab) in Crohn's disease: systematic review and meta-analysis
}

\author{
Paweł Kawalec ${ }^{1}$, Alicja Mikrut², Natalia Wiśniewska², Andrzej Pilc ${ }^{3}$
}

${ }^{1}$ Institute of Public Health, Collegium Medicum, Jagiellonian University, Cracow, Poland ${ }^{2}$ Centrum HTA Sp. z o.o., Sp. komandytowa, Cracow, Poland

3Institute of Pharmacology, Polish Academy of Sciences, Cracow, Poland

Submitted: 29 December 2012

Accepted: 21 March 2013

Arch Med Sci 2013; 9, 5: 765-779

DOI: $10.5114 /$ aoms.2013.38670

Copyright @ 2013 Termedia \& Banach

\begin{abstract}
Introduction: This meta-analysis compares the effectiveness and safety of tumor necrosis factor $\alpha$ (TNF- $\alpha$ ) antibodies (infliximab, adalimumab and certolizumab) with either a placebo or each of them in the treatment of Crohn's disease (CD). Material and methods: A systematic review of literature published up to November 2012 was performed and a meta-analysis of identified studies was carried out. We searched the following databases: PubMed, EMBASE, The Cochrane Library and others. Only randomized or clinical controlled trials were included. Results: Nineteen clinical trials fulfilled the established criteria ( 5 studies for infliximab vs. placebo, 6 for each adalimumab or certolizumab vs. placebo and 2 comparing infliximab with adalimumab). The results of meta-analysis showed that anti-TNF therapy in patients with CD is safe and statistically significantly more effective when compared with the placebo for induction of remission at week $4(\mathrm{RB}=1.90,95 \% \mathrm{Cl}: 1.55-2.33, p<0.00001)$, maintenance of remission at weeks $20-30(\mathrm{RB}=1.86,95 \% \mathrm{Cl}: 1.61-2.15, p<0.00001)$ and at weeks $48-56$ $(\mathrm{RB}=2.75,95 \% \mathrm{Cl}: 2.13-3.54, p<0.00001)$ in patients who responded to the induction therapy and patients randomized before the induction. Anti-TNF agents were also superior to the placebo in fistula healing (during short-term induction, as well as long-term maintenance) and inducing CR-70 but not CR-100 at week 4. Moreover, the anti-TNF therapy had a significant effect on achieving both CR-70 and CR-100 during long-term maintenance.

Conclusions: Infliximab, adalimumab and certolizumab are effective as both induction and maintenance therapy in moderate to severe Crohn's disease in adults, including patients with fistulas. The safety profile was acceptable.
\end{abstract}

Key words: Crohn's disease, tumor necrosis factor- $\alpha$ antibodies, systematic review, meta-analysis.

\section{Introduction}

Crohn's disease (CD) is a chronic and disabling inflammatory disease of the digestive tract of unknown etiology. It may affect any part of the gastrointestinal tract from the mouth to the anus, but most commonly the terminal ileum or the ileocecal region is affected. The highest incidence of ulcerative colitis and CD has been reported in: Northern Europe, the United Kingdom and North America [1]. The etiology of CD remains unknown, but the characteristic disproportionate inflammatory response

\author{
Corresponding author: \\ Paweł Kawalec MD, PhD \\ Jagiellonian University \\ 20 Grzegorzecka St \\ 31-531 Cracow, Poland \\ Phone: +48 607345792 \\ E-mail: \\ pawel.kawalec@uj.edu.pl
}


in the gut may develop through various mechanisms at the cellular and subcellular level [1]. Current medical treatment of $C D$, besides conventional therapy such as sulfasalazine, 5-aminosalicylates, corticosteroids and immunomodulators, include biological therapy [2]. Tumor necrosis factor (TNF) $\alpha$ is a crucial mediator of the abnormal immune response in the gut and in recent years, biological therapies targeting TNF- $\alpha$ have significantly improved the management of $C D$ refractory to conventional therapies [3]. Infliximab, adalimumab and certolizumab pegol are anti-TNF- $\alpha$ antibodies approved for the treatment of CD.

The aim of this systematic review with metaanalysis was to assess the effectiveness and safety of infliximab, adalimumab and certolizumab when compared with placebo (or each other) for induction and maintenance treatment, as well as fistula closure in adults with Crohn's disease.

\section{Material and methods}

This meta-analysis included all parallel randomized or clinical controlled trials (CCTs) comparing infliximab, adalimumab or certolizumab in any dosage/treatment regimen with placebo or each other. The studies included had to be performed with a control group but not necessarily with random allocation and/or blinding. Articles published as a full text were preferable because of the opportunity to verify the reliability or potential usefulness of the studies (availability of the full text of primary studies allows an accurate assessment of their quality and also provides essential information about the study population, applied treatment regimen and specific data to extract), but unpublished ones were also taken into consideration. The participants in these studies were adults $(\geq 18$ years old) with moderate to severe, as well as fistulizing, active CD. Studies concerning combination therapy in comparison with monotherapy or surgical intervention, or comparing different dosage/treatment regimens of the same drug, were excluded.

In order to find the primary studies, the following electronic databases were searched up to November 2012: MEDLINE via PubMed, EMBASE and the Cochrane Central Register of Controlled Trials. Searches for primary studies were also restricted and based on appropriate MeSH and EMTREE terms that meet the requirements: Crohn's disease and the intervention infliximab, adalimumab or certolizumab, combined with Boole's logical operators (Table I). The Cochrane Database of Systematic Reviews, DARE, PubMed and EMBASE databases were also searched for review articles. In addition, the Cochrane IBD/FBD Review Group Specialized Trials Register and websites of the British Society of Gastroenterology and European Crohn's and Colitis Organization were searched for relevant information concerning the clinical trials. Registers of controlled trials (www.clinicaltrials.gov) were searched for unpublished or ongoing trials. Reference lists from potentially relevant papers were hand-searched in order to identify additional studies.

The methods of the systematic reviews were applied as described in the Cochrane Handbook [4], and the report was prepared according to the preferred reporting items for systematic reviews and meta-analyses (PRISMA) guidelines [5]. The search and selection of the trials that were included were conducted by two reviewers independently. All of the potentially relevant articles were retained and their full text was critically reviewed according to the predefined inclusion or exclusion criteria. Data extraction was performed by a single reviewer using a standard data extraction form, before being then checked by a second reviewer. For the included studies, information was extracted on study design, severity of disease, dosage and scheme of

Table I. MeSH subject headings and EMTREE keywords used in constructed search strategy for primary studies (last updated: 22.11.2012)

\begin{tabular}{|c|c|}
\hline \multicolumn{2}{|r|}{ Key words (combined with Boole's logical operator, OR) } \\
\hline Medical condition & $\begin{array}{l}\text { Crohn disease OR Crohn's disease OR Crohns disease OR Ileocolitis OR Ileitis, Terminal OR Ileitis, } \\
\text { regional OR Colitis, granulomatous OR Enteritis, granulomatous OR Enteritis, regional OR Enteritis } \\
\text { regionalis OR Regional enterocolitis OR Morbus Crohn OR Cleron disease }\end{array}$ \\
\hline \multirow[t]{3}{*}{ Intervention } & $\begin{array}{l}\text { Infliximab OR Remicade OR Revellex OR Avakine OR Monoclonal antibody cA2 OR MAb CA2 OR } \\
\text { Schering-Plough brand of infliximab OR Schering brand of infliximab OR Centocor brand } \\
\text { of infliximab OR Essex brand of infliximab }\end{array}$ \\
\hline & Adalimumab OR Humira OR trudexa OR D2E7 antibody OR Abbott brand of adalimumab \\
\hline & $\begin{array}{l}\text { Certolizumab OR Cimzia OR CDP870 OR CDP } 870 \text { OR UCB Pharma brand of certolizumab pegol OR } \\
\text { Pegylated tumor necrosis factor alpha antibody Fab fragment OR pha } 738144 \text { OR pha738144 }\end{array}$ \\
\hline $\begin{array}{l}\text { Methodological } \\
\text { limits }\end{array}$ & $\begin{array}{l}\text { PubMed: Humans, Randomized Controlled Trial, Clinical Trials, Controlled Clinical Trial } \\
\text { EMBASE: Humans, Controlled Clinical Trials, Randomized Controlled Trial, Embase only } \\
\text { CENTRAL: No limits applied; word variations have been searched }\end{array}$ \\
\hline Language limits & PubMed, EMBASE: English, French, German, Polish \\
\hline
\end{tabular}


the administered anti-TNF- $\alpha$ agents, duration of treatment and clinical outcomes. The methodological quality of each of the trials included was assessed independently by two reviewers. Randomized clinical trials (RCTs) included in the present review were evaluated using the Jadad scale [6], and the CCT was assessed using the NewcastleOttawa Scale (NOS) [7].

The outcome measures reported in the selected studies were based mainly on changes in the Crohn's Disease Activity Index (CDAI) and included: clinical response defined as a reduction of $\geq 70$ points (CR-70) or of $\geq 100$ points (CR-100) from the baseline in the CDAI score, and clinical remission defined as a decrease in the CDAl score of $\leq 150$ points from baseline. Safety profile was also assessed.

\section{Statistical analysis}

For dichotomous data, the impact of the intervention was expressed as a relative benefit - RB (described as risk ratio (RR) on the graphs presented due to specific labeling of the effect measurement in the statistical analysis software) with $95 \%$ confidence intervals $(\mathrm{Cl})$. Adverse events were summarized with RR (relative risk). The results obtained from separate trials were combined using appropriate meta-analysis methods. The inverse variance, Mantel-Haenszel or Der Simonian-Laird effects model was used according to the data input and heterogeneity test results. The results were pooled for sufficiently similar outcomes and homogeneous data (determined by the degree of clinical and statistical heterogeneity). The clinical heterogeneity was assessed by examining the characteristics of the featured studies, whereas the statistical heterogeneity was assessed using the $\chi^{2}$ test, with the significance regarded as a $p$ value $=0.10$. Relative parameters were calculated using a fixed effects model when the statistical heterogeneity was not detected, and a random effects model was used when heterogeneity was present. In all of the analyses RevMan 5.1.0 was used.

\section{Results}

\section{Systematic review}

The initial search identified 1072 citations, 1025 of which were excluded after examining the titles and abstracts. A further performed selection resulted in the identification of 47 potentially eligible studies. A total of 28 articles were excluded for various reasons, and 18 RCTs and 1 CCT fulfilled the inclusion criteria (Figure 1). In addition, 45 ongoing trials of infliximab, adalimumab or certolizumab were identified at www.clinicaltrials.gov, although they did not meet the inclusion criteria.

The studies included two trial designs: induction therapy and maintenance therapy, in both populations of adult patients: moderate to severe, predominantly or wholly non-fistulizing CD and fistulizing disease. All the studies were published in English as peer-reviewed articles. Among the five included randomized controlled trials comparing infliximab with placebo, 3 RCTs were conducted in

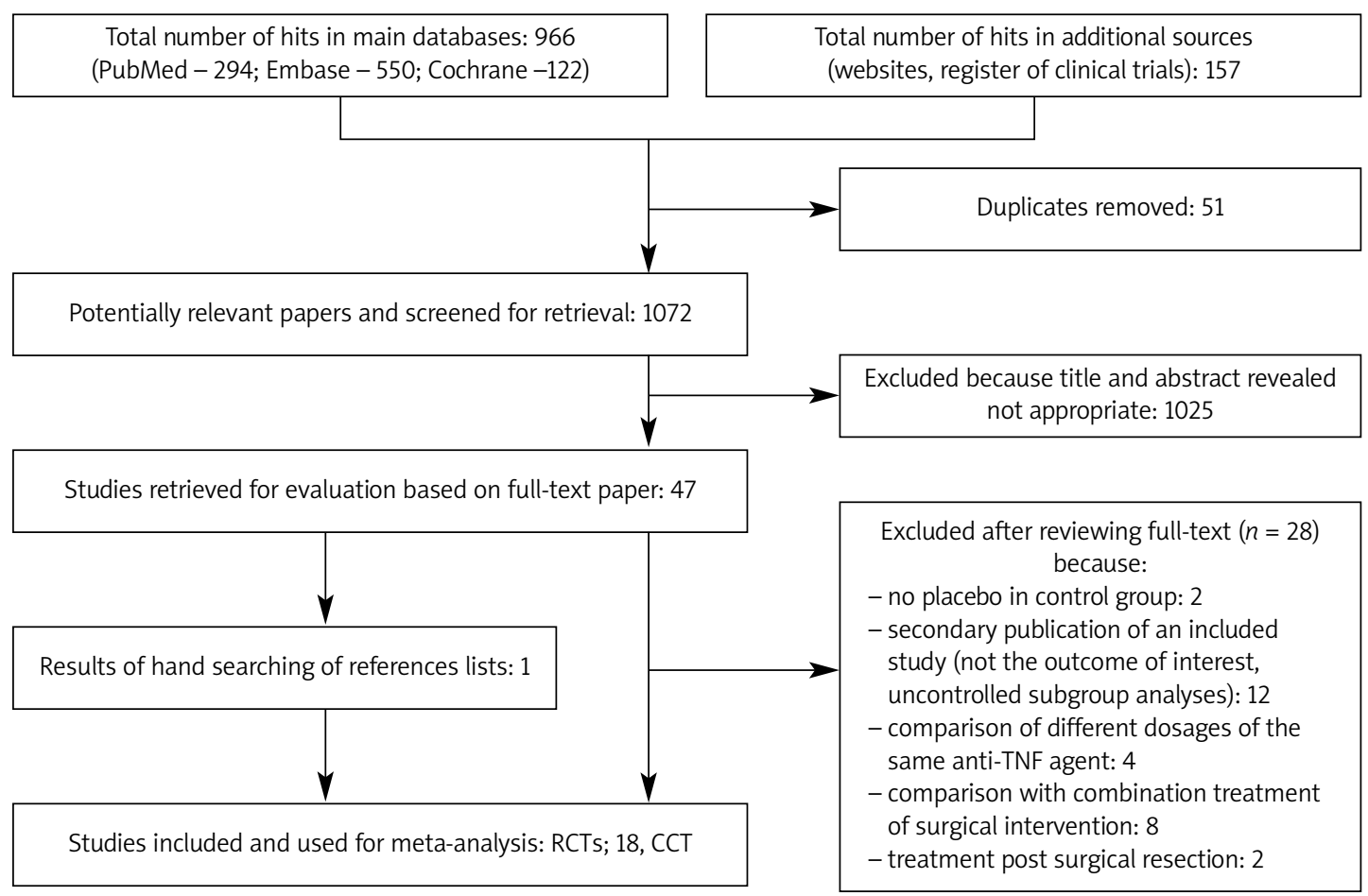

Figure 1. PRISMA flow diagram for selection of studies identified in the systematic review 
patients with non-fistulizing Crohn's disease for induction treatment. The characteristics and the methodological quality of the trials included are described in Table II.

Each of the studies included had a parallel group design and almost all of the RCTs were conducted with double blinding. A majority of the randomized studies scored $\geq 3$ points on the Jadad scale, indicating good methodological quality, except for study [26] which scored two points due to its open-label design, and studies $[18,22]$ due to a lack of description of withdrawals and dropouts. Six of the studies did not provide information on allocation concealment (Table III).

\section{Efficacy analysis}

We performed a meta-analysis of eligible RCTs that compared infliximab, adalimumab and certolizumab (separately as subgroups and then together) with a placebo. In particular comparisons, combined doses of anti-TNF agents were used. The efficacy endpoints were: clinical remission at week 4 for short- and one long-term induction trials and separately at weeks 20-30, for studies evaluating the maintenance of remission after an open-label induction (primarily among responders) at weeks 48-56. Week 4 was chosen because in most of the induction studies the results were shown for that period, and because the placebo response and remission rates increased after 4 weeks in the induction studies [8]. An evaluation of the clinical response (CR-70 or CR-100) was performed separately for both short- and long-term induction studies (with the data extracted at week 4) and for longterm maintenance studies (48-56 weeks, one study [24] lasted only 26 weeks). For the fistulizing form of $C D$, the efficacy endpoints were closure of $\geq 50 \%$ of draining fistulas and complete fistula closure after $\geq 2$ consecutive visits.

\section{Induction of remission at week 4}

Eight trials assessing the efficacy of the three anti-TNF agents for short-term induction of remission were included: seven short-term trials $[9,14$, 15, 19-22] and one long-term induction trial [23]. We included the Winter et al. 2004 [20] study, although certolizumab was administered not subcutaneously but intravenously. Finally, it turned out

Table II. Methodological quality of included RCTs and CCT

\begin{tabular}{|c|c|c|c|c|c|c|c|}
\hline \multirow[t]{2}{*}{ Study } & \multicolumn{6}{|c|}{ The Jadad scores } & \multirow{2}{*}{$\begin{array}{c}\text { Allocation } \\
\text { concealmen }\end{array}$} \\
\hline & 1 & 2 & 3 & 4 & 5 & Total Jadad & \\
\hline [9] Targan 1997 & 1 & 0 & 1 & 1 & 1 & 4 & Yes \\
\hline [10] Rutgeerts 1999 & 1 & 0 & 1 & 0 & 1 & 3 & Yes \\
\hline [11] Hanauer 2002 & 1 & 0 & 1 & 0 & 1 & 3 & Yes \\
\hline [12] Sands 2004 & 1 & 0 & 1 & 0 & 1 & 3 & Unclear \\
\hline [13] Present 1999 & 1 & 0 & 1 & 1 & 1 & 4 & Yes \\
\hline [14] Hanauer 2006 & 1 & 0 & 1 & 0 & 1 & 3 & Yes \\
\hline [15] Sandborn 2007 & 1 & 0 & 1 & 0 & 1 & 3 & Yes \\
\hline [16] Sandborn 2007 & 1 & 0 & 1 & 0 & 1 & 3 & Yes \\
\hline [17] Colombel 2007 & 1 & 0 & 1 & 0 & 1 & 3 & Yes \\
\hline [18] Rutgeerts 2012 & 1 & 0 & 1 & 0 & 0 & 2 & Unclear \\
\hline [19] Watanabe 2012 & 1 & 0 & 1 & 0 & 1 & 3 & Unclear \\
\hline [20] Winter 2004 & 1 & 0 & 1 & 0 & 1 & 3 & Unclear \\
\hline [21] Schreiber 2005 & 1 & 0 & 1 & 0 & 1 & 3 & Unclear \\
\hline [22] Sandborn 2011 & 1 & 0 & 1 & 0 & 0 & 2 & Unclear \\
\hline [23] Sandborn 2007 & 1 & 0 & 1 & 0 & 1 & 3 & Yes \\
\hline $\begin{array}{l}\text { [24] Schreiber 2007, } \\
\text { [25] Schreiber } 2011\end{array}$ & 1 & 0 & 1 & 0 & 1 & 3 & Yes \\
\hline [26] Van Assche 2012 & 1 & 0 & 0 & 0 & 1 & 2 & Yes \\
\hline Study & \multicolumn{6}{|c|}{ The NOS score } & \\
\hline [27] Riis 2012 & \multicolumn{6}{|c|}{ Good quality } & \\
\hline
\end{tabular}

1 - Was the study described as randomized?; 2 - Was the method of randomization described and appropriate?; 3 - Was the study described as double blind?; 4 - Was the method of blinding described and appropriate?; 5 - Were withdrawals and dropouts described? Additional explanation: question 2 has been scored 0 in case of lack of any description or when the method of randomization used in the study was other than simple; question 4 has been scored 0 in case of lack of any description of blinding or when the presented description was not detailed 
Table III. Characteristics of included studies

\begin{tabular}{|c|c|c|c|c|}
\hline $\begin{array}{l}\text { Study author } \\
\text { and year } \\
\text { of publication; } \\
\text { design; sites }\end{array}$ & $\begin{array}{l}\text { Population; } \\
\text { severity of CD }\end{array}$ & $N$ (for efficacy) & $\begin{array}{l}\text { Dosage and schedule, duration } \\
\text { of treatment and follow-up }\end{array}$ & $\begin{array}{l}\text { Outcomes and } \\
\text { point at which } \\
\text { extracted }\end{array}$ \\
\hline \multicolumn{5}{|c|}{ Infliximab vs. placebo } \\
\hline $\begin{array}{l}\text { [9] Targan } \\
\text { 1997; RCT; } \\
18 \text { sites }\end{array}$ & $\begin{array}{l}\text { Moderate-to- } \\
\text { severe Crohn's } \\
\text { disease }\end{array}$ & $\begin{array}{l}\text { Placebo, } n=25 ; \\
\text { infliximab } 5 \mathrm{mg}, \\
n=27 ; 10 \mathrm{mg}, n=28 \\
20 \mathrm{mg}, n=28\end{array}$ & $\begin{array}{l}\text { Induction: } \\
\text { placebo or infliximab } 5 \mathrm{mg} / \mathrm{kg} ; 10 \mathrm{mg} / \mathrm{kg} \text {, } \\
\text { or } 20 \mathrm{mg} / \mathrm{kg} \text { at week } 0 \text { (to week 12) }\end{array}$ & $\begin{array}{c}\text { Remission: } \\
\text { CDAI }<150 \text { at } \\
\text { weeks } 4 \text { and } 12\end{array}$ \\
\hline $\begin{array}{l}\text { [10] Rutgeerts } \\
\text { 1999; RCT; } \\
17 \text { sites }\end{array}$ & $\begin{array}{l}\text { Crohn's disease } \\
\text { and clinical } \\
\text { response to ini- } \\
\text { tial treatment } \\
\text { in previous } \\
\text { study [9] }\end{array}$ & $\begin{array}{l}\text { Placebo, } n=36 \\
\text { infliximab, } n=27\end{array}$ & $\begin{array}{l}\text { Maintenance: } \\
\text { initial response to placebo or infliximab } \\
\text { (Targan), then at week } 12 \text { randomiza- } \\
\text { tion to placebo or infliximab } 10 \mathrm{mg} / \mathrm{kg} \text {, } \\
\text { at 8-weekly intervals thereafter } \\
\text { (to week 48) }\end{array}$ & $\begin{array}{l}\text { Remission: } \\
\text { CDAI } \leq 150 \\
\text { at week } 48\end{array}$ \\
\hline $\begin{array}{l}\text { [11] Hanauer } \\
\text { 2002, } \\
\text { ACCENT I; } \\
\text { RCT; } 55 \text { sites }\end{array}$ & $\begin{array}{l}\text { Moderate-to- } \\
\text { severe Crohn's } \\
\text { disease }\end{array}$ & $\begin{array}{l}\text { Randomized as respon- } \\
\text { ders at week } 2 \text { : placebo, } \\
n=110 \text {; infliximab } 5 \mathrm{mg}, \\
n=113 ; \\
\text { infliximab } 5 \text { and } 10 \mathrm{mg}, \\
n=112\end{array}$ & $\begin{array}{l}\text { Maintenance: } \\
\text { initial response to open-label infliximab } \\
5 \mathrm{mg} / \mathrm{kg} \text {, then randomization to placebo } \\
\text { or infliximab } 5 \mathrm{mg} / \mathrm{kg} \text { at weeks } 2 \text { and } \\
6 \text { or infliximab } 5 \mathrm{mg} / \mathrm{kg} \text { at weeks } 2 \text { and } \\
6 \text { followed by } 10 \mathrm{mg} / \mathrm{kg} \text { at } 8 \text {-weekly } \\
\text { intervals thereafter (to week } 46 \text { ). } \\
\text { Note: both infliximab groups received } \\
5 \mathrm{mg} / \mathrm{kg} \text { at weeks } 2 \text { and } 6\end{array}$ & $\begin{array}{l}\text { Remission: } \\
\text { CDAI } \leq 150 \\
\text { at week } 54 \\
\text { (for '2-week' } \\
\text { responders) }\end{array}$ \\
\hline $\begin{array}{l}\text { [12] Sands } \\
\text { 2004, } \\
\text { ACCENT II, } \\
\text { RCT; } 45 \text { sites }\end{array}$ & $\begin{array}{l}\text { Crohn's disease } \\
\text { and } \geq 1 \text { drain- } \\
\text { ing abdominal } \\
\text { or perianal fis- } \\
\text { tulas of } \\
\geq 3 \text { months' } \\
\text { duration }\end{array}$ & $\begin{array}{l}\text { Randomized respon- } \\
\text { ders, } n=195: \text { placebo, } \\
n=99 ; \text { infliximab, } \\
n=96\end{array}$ & $\begin{array}{l}\text { Maintenance: } \\
\text { infliximab } 5 \mathrm{mg} / \mathrm{kg} \text { at weeks } 0,2 \text { and } \\
6 \text { for all patients; at week } 14 \text { responders } \\
(n=195) \text { and non-responders }(n=87) \\
\text { were randomized to placebo or inflix- } \\
\text { imab } 5 \mathrm{mg} / \mathrm{kg} \text { at } 8 \text {-weekly intervals } \\
\text { thereafter (to week } 54)\end{array}$ & $\begin{array}{l}\text { Absence of any } \\
\text { draining fistulas } \\
\text { or reduction } \geq 50 \% \\
\text { in the number of } \\
\text { draining fistulas } \\
\text { at week } 54 \text { (for } \\
\text { responders) }\end{array}$ \\
\hline $\begin{array}{l}\text { [13] Present } \\
\text { 1999; RCT; } \\
12 \text { sites }\end{array}$ & $\begin{array}{l}\text { Crohn's disease } \\
\text { and draining } \\
\text { abdominal or } \\
\text { perianal fistulas } \\
\text { of } \geq 3 \text { months' } \\
\text { duration }\end{array}$ & $\begin{array}{l}\text { Placebo, } n=31 \\
\text { infliximab } 5 \mathrm{mg}, n=31 \\
10 \mathrm{mg}, n=32\end{array}$ & $\begin{array}{l}\text { Induction: } \\
\text { placebo or infliximab } 5 \mathrm{mg} / \mathrm{kg} \text { or } \\
10 \mathrm{mg} / \mathrm{kg} \text { at weeks } 0,2 \text { and } 6 \\
\text { (to week } 18 \text { ) }\end{array}$ & $\begin{array}{l}\text { Absence of any } \\
\text { draining fistulas } \\
\text { or reduction } \geq 50 \% \\
\text { in the number of } \\
\text { draining fistulas } \\
\text { at week } 18\end{array}$ \\
\hline \multicolumn{5}{|c|}{ Adalimumab vs. placebo } \\
\hline $\begin{array}{l}\text { [14] Hanauer } \\
\text { 2006, } \\
\text { CLASSIC I; RCT; } \\
55 \text { sites }\end{array}$ & $\begin{array}{l}\text { Moderate-to- } \\
\text { severe Crohn's } \\
\text { disease }\end{array}$ & $\begin{array}{l}\text { Placebo, } n=74 \text {; adali- } \\
\text { mumab } 40 / 20 \mathrm{mg} \\
n=74,80 / 40 \mathrm{mg} \\
n=75,160 / 80 \mathrm{mg} \\
n=76\end{array}$ & $\begin{array}{l}\text { Induction: } \\
\text { placebo or adalimumab: } 40 \mathrm{mg} / 20 \mathrm{mg} \text {, } \\
80 \mathrm{mg} / 40 \mathrm{mg} \text { or } 160 \mathrm{mg} / 80 \mathrm{mg} \text { at week } \\
\text { 0/2 (to week 4) }\end{array}$ & $\begin{array}{l}\text { Remission: } \\
\text { CDAI }<150 \\
\text { at week } 4\end{array}$ \\
\hline $\begin{array}{l}\text { [15] Sandborn } \\
\text { 2007, GAIN; } \\
\text { RCT; } 52 \text { sites }\end{array}$ & $\begin{array}{l}\text { Moderate-to- } \\
\text { severe Crohn's } \\
\text { disease for } \\
\geq 4 \text { months, } \\
\text { after previous } \\
\text { infliximab }\end{array}$ & $\begin{array}{l}\text { Placebo, } n=166 \\
\text { adalimumab, } n=159\end{array}$ & $\begin{array}{l}\text { Induction: } \\
\text { placebo or adalimumab 160/80 mg } \\
\text { at week 0/2 (to week 4) }\end{array}$ & $\begin{array}{l}\text { Remission: } \\
\text { CDAl < } 150 \text { at } \\
\text { week } 4 \text {. Response: } \\
\text { decrease in CDAl } \\
\text { score of }>70 \\
\text { or }>100 \text { points } \\
\text { at week } 4\end{array}$ \\
\hline $\begin{array}{l}\text { [16] Sandborn } \\
\text { 2007, } \\
\text { CLASSIC II; } \\
\text { RCT; } 53 \text { sites }\end{array}$ & $\begin{array}{l}\text { As above; } \\
55 \text { patients in } \\
\text { remission at } \\
\text { week } 0 \text { (week } \\
4 \text { of CLASSIC I) }\end{array}$ & $\begin{array}{l}\text { Placebo, } n=18 \text {; adali- } \\
\text { mumab } 40 \mathrm{mg} \text { every } \\
\text { other week, } n=19 ; \\
\text { adalimumab } 40 \mathrm{mg} \\
\text { weekly, } n=18\end{array}$ & $\begin{array}{l}\text { Maintenance: } \\
\text { initial adalimumab or placebo as part of } \\
\text { study CLASSIC-I, then remission after } \\
\text { open-label adalimumab } 40 \mathrm{mg} \text { (week } 0 \\
\text { (week } 4 \text { of CLASSIC I) and 2), then ran- } \\
\text { domization to placebo or adalimumab } \\
40 \text { mg weekly or } 40 \text { mg every other } \\
\text { week (to week } 56 \text { ) }\end{array}$ & $\begin{array}{c}\text { Remission: } \\
\text { CDAI } \leq 150 \text { at } 56 \\
\text { weeks. Response: } \\
\text { decrease in CDAl } \\
\text { score of }>70 \text { or } \\
>100 \text { points at week } \\
56 \text { (from week } 0 \\
\text { in CLASSIC I) }\end{array}$ \\
\hline
\end{tabular}


Table III. Cont.

\begin{tabular}{|c|c|c|c|c|}
\hline $\begin{array}{l}\text { Study author } \\
\text { and year } \\
\text { of publication; } \\
\text { design; sites }\end{array}$ & $\begin{array}{c}\text { Population; } \\
\text { severity of } C D\end{array}$ & $N$ (for efficacy) & $\begin{array}{l}\text { Dosage and schedule, duration } \\
\text { of treatment and follow-up }\end{array}$ & $\begin{array}{l}\text { Outcomes and } \\
\text { point at which } \\
\text { extracted }\end{array}$ \\
\hline $\begin{array}{l}\text { [17] Colombel } \\
\text { 2007, CHARM; } \\
\text { RCT, } 92 \text { sites }\end{array}$ & $\begin{array}{l}\text { Moderate-to- } \\
\text { severe, active } \\
\text { Crohn's dis- } \\
\text { ease, also with } \\
\text { draining } \\
\text { fistulas }\end{array}$ & $\begin{array}{l}\text { Randomized respon- } \\
\text { ders: placebo, } n=170 ; \\
\text { adalimumab } 40 \mathrm{mg} \\
\text { every other week, } \\
n=172 \text {; adalimumab } \\
40 \text { mg weekly, } n=157 \\
\text { with fistulas: placebo, } \\
n=47 ; \text { adalimumab, } \\
n=70\end{array}$ & $\begin{array}{l}\text { Maintenance: } \\
\text { initial response to open-label adalimum- } \\
\text { ab } 80 / 40 \mathrm{mg} \text { (week } 0 / 2 \text { ); at week } 4 \text { ran- } \\
\text { domization to placebo or adalimumab } \\
40 \mathrm{mg} \text { weekly or } 40 \mathrm{mg} \text { every other } \\
\text { week (to week } 56 \text { ) }\end{array}$ & $\begin{array}{c}\text { Remission: CDAI } \\
<150 \text { at weeks } \\
26 \text { and } 56 \text { (for } \\
\text { responders). } \\
\text { Response: } \\
\text { decrease in CDAI } \\
\text { score of > } 70 \text { or } \\
>100 \text { points at } \\
\text { weeks } 25 \text { and } 56 . \\
\text { Complete fistula } \\
\text { healing }\end{array}$ \\
\hline $\begin{array}{l}\text { [18] Rutgeerts } \\
\text { 2012, EXTEND; } \\
\text { RCT; } 19 \text { sites }\end{array}$ & $\begin{array}{l}\text { Moderate-to- } \\
\text { severe Crohn's } \\
\text { disease for } \geq 4 \\
\text { months and } \\
\text { presence of } \\
\text { mucosal ulcer- } \\
\text { ation, also with } \\
\text { fistulas (12\%) }\end{array}$ & $\begin{array}{l}\text { Placebo, } n=65 \\
\text { adalimumab, } n=64\end{array}$ & $\begin{array}{l}\text { Maintenance: } \\
\text { initial response to open-label adalimum- } \\
\text { ab 160/80 mg at week 0/2; at week } 4 \\
\text { randomization to placebo or adalimumab } \\
40 \text { mg every other week (to week 52). } \\
\text { Note: maintenance without preselection } \\
\text { for a response to induction but randomiza- } \\
\text { tion stratified by CR-70 status at week } 4\end{array}$ & $\begin{array}{c}\text { Remission: } \\
\text { CDAl < } 150 \text { at } \\
\text { weeks } 12 \text { and } 52 . \\
\text { Response: } \\
\text { decrease in CDAl } \\
\text { score of }>70 \text { or } \\
>100 \text { points at } \\
\text { weeks } 12 \text { and } 52\end{array}$ \\
\hline \multirow[t]{2}{*}{$\begin{array}{l}\text { [19] Watanabe } \\
\text { 2012; RCT; } \\
\text { multicentre }\end{array}$} & $\begin{array}{l}\text { Moderate-to- } \\
\text { severe, active } \\
\text { Crohn's dis- } \\
\text { ease for } \geq 4 \\
\text { months }\end{array}$ & $\begin{array}{l}\text { Induction: placebo, } \\
n=23 ; \text { adalimumab } \\
\text { 160/80 mg, } n=33 ; \\
80 / 40 \mathrm{mg}, n=34\end{array}$ & $\begin{array}{l}\text { Induction: } \\
\text { placebo or adalimumab } 160 / 80 \mathrm{mg} \text { or } \\
\text { adalimumab } 80 / 40 \mathrm{mg} \text { at week } 0 / 2 \\
\text { (to week 4) }\end{array}$ & $\begin{array}{l}\text { Remission: CDAl } \\
<150 \text { at week } 4 . \\
\text { Response: decrease } \\
\text { in CDAl score of } \\
>70 \text { or }>100 \text { points } \\
\text { at week } 2 \text { and } 4 \text { (for } \\
\text { patients with remis- } \\
\text { sion at week 2) }\end{array}$ \\
\hline & & $\begin{array}{l}\text { Maintenance: } \\
\text { placebo, } n=25 \text {; adali- } \\
\text { mumab } 40 \mathrm{mg}, n=25\end{array}$ & $\begin{array}{l}\text { Maintenance (responders only): } \\
\text { at week } 4 \text { randomization to placebo } \\
\text { or adalimumab } 40 \text { mg every other week } \\
\text { for } 52 \text { weeks }\end{array}$ & $\begin{array}{l}\text { Remission: CDAl } \\
<150 \text { at week } 52 \text {. } \\
\text { Response: as } \\
\text { above, at week } 52\end{array}$ \\
\hline \multicolumn{5}{|c|}{ Certolizumab vs. placebo } \\
\hline $\begin{array}{l}\text { [20] Winter } \\
\text { 2004; RCT; } \\
24 \text { sites }\end{array}$ & $\begin{array}{l}\text { Moderate to } \\
\text { severe Crohn's } \\
\text { disease }\end{array}$ & $\begin{array}{l}\text { Placebo, } n=24 \\
\text { certolizumab } 1.25 \mathrm{mg}, \\
n=2 ; 5 \mathrm{mg}, n=26 \\
10 \mathrm{mg}, n=17 \\
20 \mathrm{mg} / \mathrm{kg}, n=23\end{array}$ & $\begin{array}{l}\text { Induction: } \\
\text { placebo or certolizumab } 5 \mathrm{mg} / \mathrm{kg} ; 10 \mathrm{mg} \\
\text { or } 20 \mathrm{mg} / \mathrm{kg} \text { at week } 0 \text { (followed to } \\
\text { week } 12 \text { ) }\end{array}$ & $\begin{array}{c}\text { Remission: CDAl } \\
\leq 150 \text { at week } 4 . \\
\text { Response: } \\
\text { decrease in CDAl } \\
\text { score of } \geq 100 \\
\text { points at week } 4\end{array}$ \\
\hline $\begin{array}{l}\text { [21] Schreiber } \\
\text { 2005; RCT; } \\
58 \text { sites }\end{array}$ & $\begin{array}{l}\text { Moderate to } \\
\text { severe Crohn's } \\
\text { disease }\end{array}$ & $\begin{array}{l}\text { Placebo, } n=73 \text {; } \\
\text { certolizumab } 100 \mathrm{mg} \\
n=74 ; 200 \mathrm{mg}, n=72 ; \\
400 \mathrm{mg}, n=72\end{array}$ & $\begin{array}{l}\text { Induction: } \\
\text { placebo or certolizumab } 100 \mathrm{mg} \text {, } \\
200 \mathrm{mg} \text { or } 400 \mathrm{mg} \text { at week } 0 \text { and fur- } \\
\text { ther doses of the same medication at } \\
\text { weeks } 4 \text { and } 8\end{array}$ & $\begin{array}{l}\text { Remission: CDAl } \\
\leq 150 \text { at week } 12 \text {. } \\
\text { Response: } \\
\text { decrease in CDAl } \\
\text { score of } \geq 100 \\
\text { points }\end{array}$ \\
\hline $\begin{array}{l}\text { [22] Sandborn } \\
\text { 2011; } R C T ; \\
120 \text { sites }\end{array}$ & $\begin{array}{l}\text { Moderate to } \\
\text { severe Crohn's } \\
\text { disease }\end{array}$ & $\begin{array}{l}\text { Placebo, } n=209 \\
\text { certolizumab, } n=215\end{array}$ & $\begin{array}{l}\text { Induction: } \\
\text { placebo or certolizumab } 400 \mathrm{mg} \text { at } \\
\text { weeks } 0,2 \text {, and } 4 \text { (followed to week 6) }\end{array}$ & $\begin{array}{l}\text { Remission, CDAl } \\
\leq 150 \text { at weeks 2, } \\
4 \text { and } 6 \text {. Response: } \\
\text { decrease in CDAl } \\
\text { score of } \geq 100 \\
\text { points at weeks } 2 \text {, } \\
4 \text { and } 6\end{array}$ \\
\hline $\begin{array}{l}\text { [23] Sandborn } \\
\text { 2007, } \\
\text { PRECiSE I; } \\
\text { RCT; } 171 \text { sites }\end{array}$ & $\begin{array}{l}\text { Moderate to } \\
\text { severe, active } \\
\text { Crohn's dis- } \\
\text { ease for } \geq 3 \\
\text { months, also } \\
\text { with fistulas }\end{array}$ & $\begin{array}{l}\text { Placebo, } n=328 \\
\text { certolizumab, } n=331\end{array}$ & $\begin{array}{l}\text { Short- and long-term induction: placebo } \\
\text { or certolizumab } 400 \text { mg at weeks } 0,2 \text {, } \\
\text { and } 4 \text { and then every } 4 \text { weeks } \\
\text { (to week 26). } \\
\text { Note: treatment after week } 4 \text { without pre- } \\
\text { selection for a response to induction }\end{array}$ & $\begin{array}{l}\text { Remission: CDAl } \\
\leq 150 \text { at week } 6 \\
\text { and } 26 \text {. Response: } \\
\text { decrease in CDAl } \\
\text { score of } \geq 100 \text { points } \\
\text { at week } 6 \text { and } 26 \\
\text { (and combined) }\end{array}$ \\
\hline
\end{tabular}


Table III. Cont.

\begin{tabular}{|c|c|c|c|c|}
\hline $\begin{array}{l}\text { Study author } \\
\text { and year } \\
\text { of publication; } \\
\text { design; sites }\end{array}$ & $\begin{array}{l}\text { Population; } \\
\text { severity of } C D\end{array}$ & $N$ (for efficacy) & $\begin{array}{l}\text { Dosage and schedule, duration } \\
\text { of treatment and follow-up }\end{array}$ & $\begin{array}{c}\text { Outcomes and } \\
\text { point at which } \\
\text { extracted }\end{array}$ \\
\hline $\begin{array}{l}\text { [24] Schreiber } \\
\text { 2007, } \\
\text { PRECiSE II; } \\
\text { RCT; } 147 \text { sites }\end{array}$ & $\begin{array}{l}\text { Moderate to } \\
\text { severe, active } \\
\text { Crohn's dis- } \\
\text { ease for } \geq 3 \\
\text { months }\end{array}$ & $\begin{array}{l}\text { Placebo, } n=210 \\
\text { certolizumab, } n=215\end{array}$ & $\begin{array}{l}\text { Maintenance: } \\
\text { initial response to open label cer- } \\
\text { tolizumab at weeks } 0,2 \text {, and } 4 \text {; at week } \\
6 \text { randomization to placebo or certolizu- } \\
\text { mab } 400 \text { mg every } 4 \text { weeks (to week } \\
24 \text { and followed through week } 26 \text { ) }\end{array}$ & $\begin{array}{l}\text { Remission: } \\
\text { CDAI } \leq 150 \text {. } \\
\text { Response: } \\
\text { decrease in CDAl } \\
\text { score of } \geq 100 \\
\text { points at week } 26\end{array}$ \\
\hline $\begin{array}{l}\text { [25] Schreiber } \\
\text { 2011, } \\
\text { PRECiSE II }\end{array}$ & $\begin{array}{l}\text { As above; } \\
\text { subgroup of } \\
\text { patients with } \\
\text { draining fistu- } \\
\text { las at baseline }\end{array}$ & $\begin{array}{l}\text { Placebo, } n=30 \\
\text { certolizumab, } n=28\end{array}$ & $\begin{array}{l}\text { At week } 6 \text {, responders with draining } \\
\text { fistulas }(n=58) \text { were randomized to } \\
\text { placebo or certolizumab } 400 \text { mg every } \\
4 \text { weeks across weeks } 8-24\end{array}$ & $\begin{array}{l}\text { Absence of any } \\
\text { draining fistulas } \\
\text { at week } 26\end{array}$ \\
\hline \multicolumn{5}{|c|}{ Infliximab vs. adalimumab } \\
\hline $\begin{array}{l}\text { [26] Van Ass- } \\
\text { che } 2012 \text {, } \\
\text { SWICH; RCT; } \\
\text { single-centre }\end{array}$ & $\begin{array}{l}\text { Crohn's dis- } \\
\text { ease and ongo- } \\
\text { ing response to } \\
\text { at least } 6 \\
\text { months of } \\
\text { maintenance } \\
\text { infliximab }\end{array}$ & $\begin{array}{l}\text { Infliximab, } n=37 \\
\text { adalimumab, } n=36\end{array}$ & $\begin{array}{l}\text { Maintenance: } \\
\text { infliximab } 5 \mathrm{mg} / \mathrm{kg} \text { or adalimumab } \\
80 \mathrm{mg} \text { at baseline followed by } 40 \mathrm{mg} \\
\text { every other week for } 54 \text { weeks }\end{array}$ & $\begin{array}{l}\text { Dose intensifica- } \\
\text { tion, early treat- } \\
\text { ment termination, } \\
\text { preferences, } \\
\text { increase in the } \\
\text { CDAl of > } 100 \\
\text { above baseline }\end{array}$ \\
\hline $\begin{array}{l}\text { [27] Riis 2012; } \\
\text { retrospective, } \\
\text { cohort study }\end{array}$ & $\begin{array}{l}\text { Crohn's } \\
\text { disease }\end{array}$ & $\begin{array}{l}\text { Infliximab, } n=64 ; \\
\text { adalimumab, } n=19\end{array}$ & $\begin{array}{l}\text { Infliximab } 5 \mathrm{mg} / \mathrm{kg} \text { at weeks } 0,2 \text { and } \\
6 \text { or adalimumab } 80 \mathrm{mg} \text { at baseline } \\
\text { followed by } 40 \mathrm{mg} \text { every other week } \\
\text { (up to median } 11.4 \text { months) }\end{array}$ & $\begin{array}{l}\text { Discontinuation of } \\
\text { anti-TNF therapy, } \\
\text { hospitalization } \\
\text { and surgery rates }\end{array}$ \\
\hline
\end{tabular}

that this way of administering certolizumab did not affect the results. The overall analysis revealed that the anti-TNF therapy was significantly more effective than placebo for induction of remission at week $4(\mathrm{RB}=1.90,95 \% \mathrm{Cl}: 1.55-2.33, p<0.00001)$. Results of the analysis in subgroups have shown that the probability of remission was significantly higher for both adalimumab and certolizumab when compared to the placebo (Figure 2). A subgroup analysis could not be performed for infliximab because only one study was available for this endpoint.

\section{Maintenance of remission after open-label induction}

Seven trials that evaluated maintenance of infliximab [10, 11], adalimumab [16-19] and certolizum$a b[23,24]$ were included in the evaluation of maintenance of remission among responders after open-label induction (except for study [18] where patients underwent maintenance treatment irrespective of the response to the previous induction phase). In the overall analysis the anti-TNF therapy was significantly more effective than the placebo for the maintenance of remission at weeks 48$56(\mathrm{RB}=2.75,95 \% \mathrm{Cl}: 2.13-3.54, p<0.00001)$. In the subgroup analysis there were also significant benefits in favor of infliximab and adalimumab (Figure 3). The inclusion of the certolizumab results from study [24] did not affect the overall efficacy of the anti-TNF therapy for the maintenance of remission ( $\mathrm{RB}=2.30,95 \% \mathrm{Cl}: 1.73-3.06, p<0.00001)$. The results of subgroups (infliximab vs. placebo $R B=$ 2.00, 95\% Cl: 1.44-2.77, $p<0.0001$; adalimumab vs. placebo RB = 2.24, 95\% Cl: 1.69-2.98, $p<0.00001$; certolizumab vs. placebo $\mathrm{RB}=1.64,95 \% \mathrm{Cl}$ : $1.36-1.99, p<0.00001)$ and the results of the overall meta-analysis ( $\mathrm{RB}=1.86,95 \% \mathrm{Cl}: 1.61-2.15$, $p<0.00001)$ for the maintenance of remission at weeks 20-30 (including study [23] with randomization before the induction) show that each of the three anti-TNF agents was significantly more effective than the placebo.

\section{Rates of fistula closure}

Seven studies evaluated the anti-TNF agents for the treatment of fistulizing CD, although only 2 of the trials were designed specifically to address this issue $[12,13]$. During short-term induction treatment with infliximab [13] and adalimumab [14, 15] significantly more patients achieved $\mathrm{a} \geq 50 \%$ reduction in fistulas in comparison with the placebo $(\mathrm{RB}=1.70,95 \% \mathrm{Cl}$ : $1.03-2.82, p=0.04)$. Similarly, in the long-term maintenance, both infliximab [12] and certolizumab [25] were associated with significantly greater rates for $\geq 50 \%$ fistula closure in the reported follow-up (RB = $1.84,95 \% \mathrm{Cl}: 1.26-2.67, p=0.001)$. When evaluating complete fistula closure, we also took into account the data from the short- and long-term induction tri- 


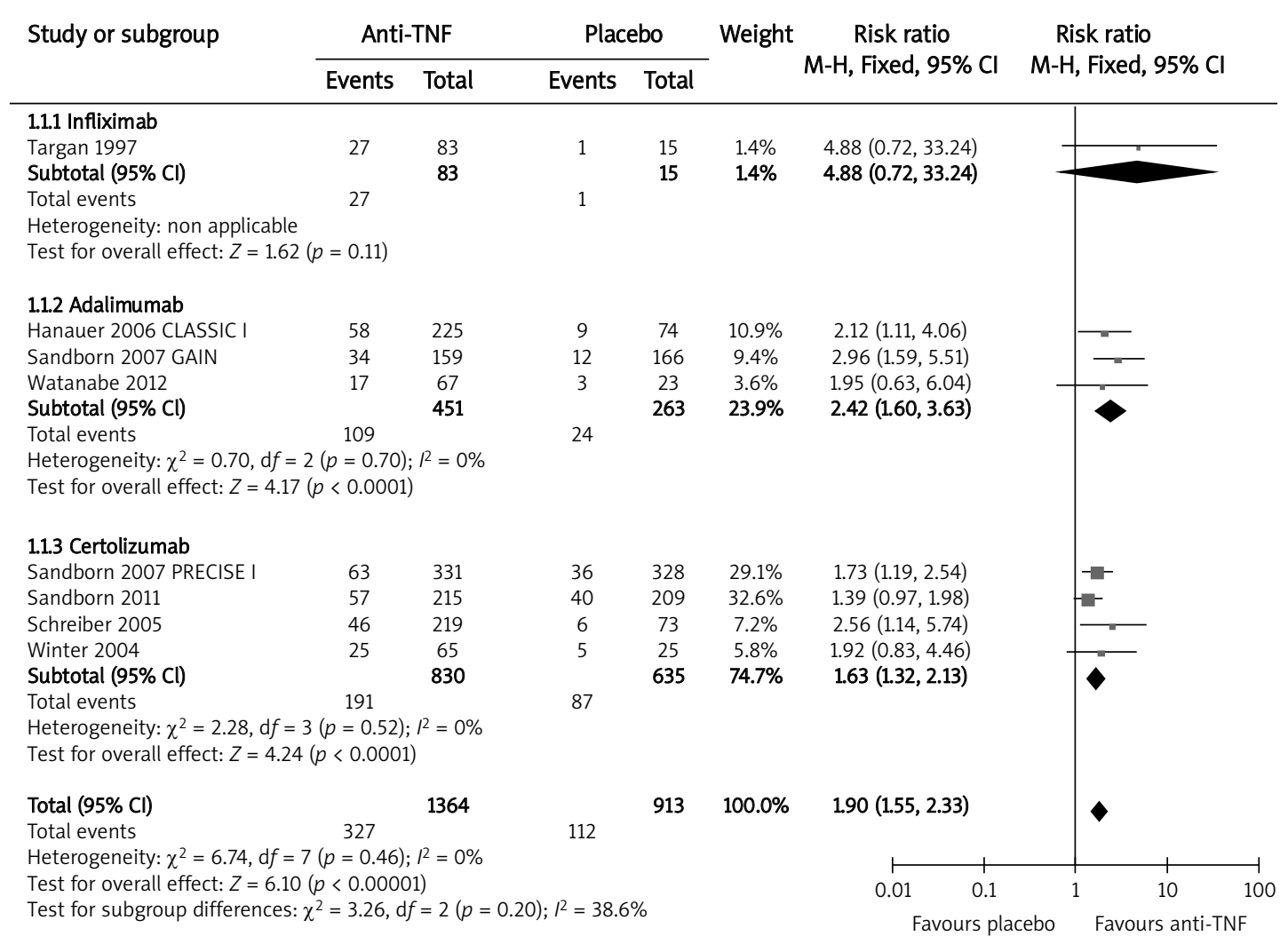

Figure 2. The effect of anti-TNF agents compared with placebo on induction of remission at week 4

\begin{tabular}{|c|c|c|c|c|c|c|c|c|c|}
\hline \multirow[t]{2}{*}{ Study or subgroup } & \multicolumn{2}{|c|}{ Anti-TNF } & \multicolumn{2}{|c|}{ Placebo } & \multirow[t]{2}{*}{ Weight } & \multirow{2}{*}{$\begin{array}{c}\text { Risk ratio } \\
\mathrm{M}-\mathrm{H} \text {, Fixed, } 95 \% \mathrm{Cl}\end{array}$} & \multirow{2}{*}{\multicolumn{2}{|c|}{$\begin{array}{l}\text { Risk ratio } \\
\mathrm{M}-\mathrm{H} \text {, Fixed, } 95 \% \mathrm{Cl}\end{array}$}} & \\
\hline & Events & Total & Events & Total & & & & & \\
\hline \multicolumn{10}{|l|}{ 1.2.1 Infliximab } \\
\hline Hanauer 2002 ACCENT I & 75 & 225 & 15 & 110 & $27.9 \%$ & $2.44(1.47,4.05)$ & & - & \\
\hline Rutgeerts 1999 & 13 & 37 & 7 & 36 & $9.8 \%$ & $1.81(0.82,4.01)$ & & & \\
\hline Subtotal $(95 \% \mathrm{Cl})$ & & 262 & & 146 & $37.7 \%$ & $2.28(1.48,3.50)$ & & & \\
\hline Total events & 88 & & 22 & & & & & & \\
\hline \multicolumn{10}{|c|}{$\begin{array}{l}\text { Heterogeneity: } \chi^{2}=0.40, \mathrm{~d} f=1(p=0.53) ; l^{2}=0 \% \\
\text { Test for overall effect: } Z=3.76(p=0.0002)\end{array}$} \\
\hline \multicolumn{10}{|l|}{ 1.2.2 Adalimumab } \\
\hline Colombel 2007 CHARM & 126 & 329 & 20 & 170 & $36.5 \%$ & $3.26(2.11,5.02)$ & & $-\square-$ & \\
\hline Rutgeerts 2012 & 21 & 64 & 6 & 65 & $8.2 \%$ & $3.55(1.54,8.23)$ & & $\square$ & \\
\hline Sandborn 2007 CLASSIC II & 30 & 37 & 8 & 18 & $14.9 \%$ & $1.82(1.06,3.13)$ & & - & \\
\hline Watanabe 2012 & 10 & 25 & 2 & 25 & $2.8 \%$ & $5.00(1.22,20.55)$ & & 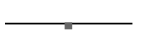 & \\
\hline Subtotal $(95 \% \mathrm{Cl})$ & & 455 & & 278 & $62.3 \%$ & $3.03(2.21,4.16)$ & & & \\
\hline Total events & 187 & & 36 & & & & & & \\
\hline \multicolumn{10}{|c|}{$\begin{array}{l}\text { Heterogeneity: } \chi^{2}=4.13, \mathrm{~d} f=3(p=0.25) ; R^{2}=27 \% \\
\text { Test for overall effect: } Z=6.87(p<0.00001)\end{array}$} \\
\hline Total $(95 \% \mathrm{Cl})$ & & 717 & & 424 & $100.0 \%$ & $2.75(2.13,3.54)$ & & - & \\
\hline Total events & 275 & 58 & & & & & & & \\
\hline \multicolumn{5}{|c|}{ Heterogeneity: $\chi^{2}=5.12, \mathrm{~d} f=5(p=0.40) ; p^{2}=2 \%$} & & & & & \\
\hline \multicolumn{5}{|c|}{ Test for overall effect: $Z=7.80\left(\chi^{2}<0.00001\right)$} & & 0.01 & 1 & 10 & 100 \\
\hline \multicolumn{5}{|c|}{ Test for subgroup differences: $\chi^{2}=1.10, \mathrm{~d} f=1(p=0.29) ; R^{2}=9.2 \%$} & & Favours pla & cebo & Favours anti-T & \\
\hline
\end{tabular}

Figure 3. The effect of anti-TNF agents compared with placebo on maintenance of remission at week 48-56 (excluding study [24])

al [23]. In the short-term induction therapy, combined infliximab [13] and adalimumab [14, 15] were superior to the placebo in the complete healing of fistulas $(\mathrm{RB}=2.44,95 \% \mathrm{Cl}: 1.13-5.28, p=0.02)$. Likewise, there was a statistically significant benefit of combined infliximab [12], adalimumab [17] and certolizumab [25] over the placebo for complete fistula closure during the maintenance treatment $(\mathrm{RB}=2.03,95 \% \mathrm{Cl}: 1.38$ $2.98, p=0.0003)$. No significant difference was detected in the rates of complete fistula healing in the 
short- and long-term induction trial [23] $(\mathrm{RB}=1.02$, 95\% Cl: 0.58-1.82, $p=0.94$ ) (Figure 4).

\section{Induction of response (70-CR and 100-CR) at week 4}

Data on the clinical response rate defined as 70-CR or 100-CR in the CDAl score at week 4 of short- and long-term induction trials were available and extracted from seven studies (one evaluating infliximab [9] and three for both adalimumab [14, $15,19]$ and certolizumab $[20,22,23])$. In the overall analysis the anti-TNF therapy was more effective than the placebo for achieving the clinical response described as 70-CR at week $4\left(\mathrm{RB}_{\text {fixed }}=1.64\right.$, $95 \% \mathrm{Cl}: 1.37-1.95, p<0.00001)$. The subgroup analysis also favored infliximab and adalimumab but not the certolizumab used in the single eligible study [23]. Data on the clinical response 100-CR at week 4 of the induction treatment was available in six trials. There were no data on infliximab and 100-CR at week 4. In the subgroup and overall analysis, there were no statistically significant differences between anti-TNF agents, both adalimumab and certolizumab versus placebo $(\mathrm{RB}=1.21,95 \% \mathrm{Cl}$ : 0.81-1.80, $p=0.35$ ) (Figure 5).

\section{Clinical response (70-CR and 100-CR) during maintenance}

Five trials evaluated the clinical response defined as 70-CR during the long-term maintenance treatment with infliximab $[10,11]$ and adalimumab [16-18]. There were no extractable data for certolizumab. The overall result of the meta-analysis demonstrated that the anti-TNF therapy was statistically beneficial when compared to the placebo in achieving 70-CR at weeks $48-56\left(\mathrm{RB}_{\text {random }}=2.06,95 \% \mathrm{Cl}: 1.32-3.23, p=0.002\right)$. To assess the $100-C R$ rates, there were no data regarding infliximab, although a study evaluating certolizumab at week 26 was included [24]. The anti-TNF therapy was associated with a significantly increased relative benefit of achieving response 100-CR during maintenance when compared to the placebo ( $R B=$ 2.03, 95\% Cl: 1.48-2.78, $p<0.0001$ ) (Figure 6). Excluding study [24], in which the data were extracted at week 26 , the final result remains statistically unaffected ( $\mathrm{RB}=2.21,95 \% \mathrm{Cl}: 1.40-3.51, p<0.0001)$.

The outcomes evaluated in 2 studies comparing infliximab and adalimumab with each other were different from those concerning placebo-controlled trials. In study [26] the results for adalimumab therapy (after switching from infliximab) were statistically inferior when compared to the continued use of infliximab with regard to the need for dose intensification, risk of early treatment termination ( $\mathrm{RR}=0.34,95 \% \mathrm{Cl}: 0.15-0.77, p=0.01)$ and risk of treatment discontinuation due to loss of response or intolerance $(\mathrm{RR}=0.10,95 \% \mathrm{Cl}$ : 0.01-0.72, $p=0.02$ ). Both important and inconsistent with the clinical results, significantly more patients preferred adalimumab over infliximab at most time points throughout the study. In the cohort study [27] most

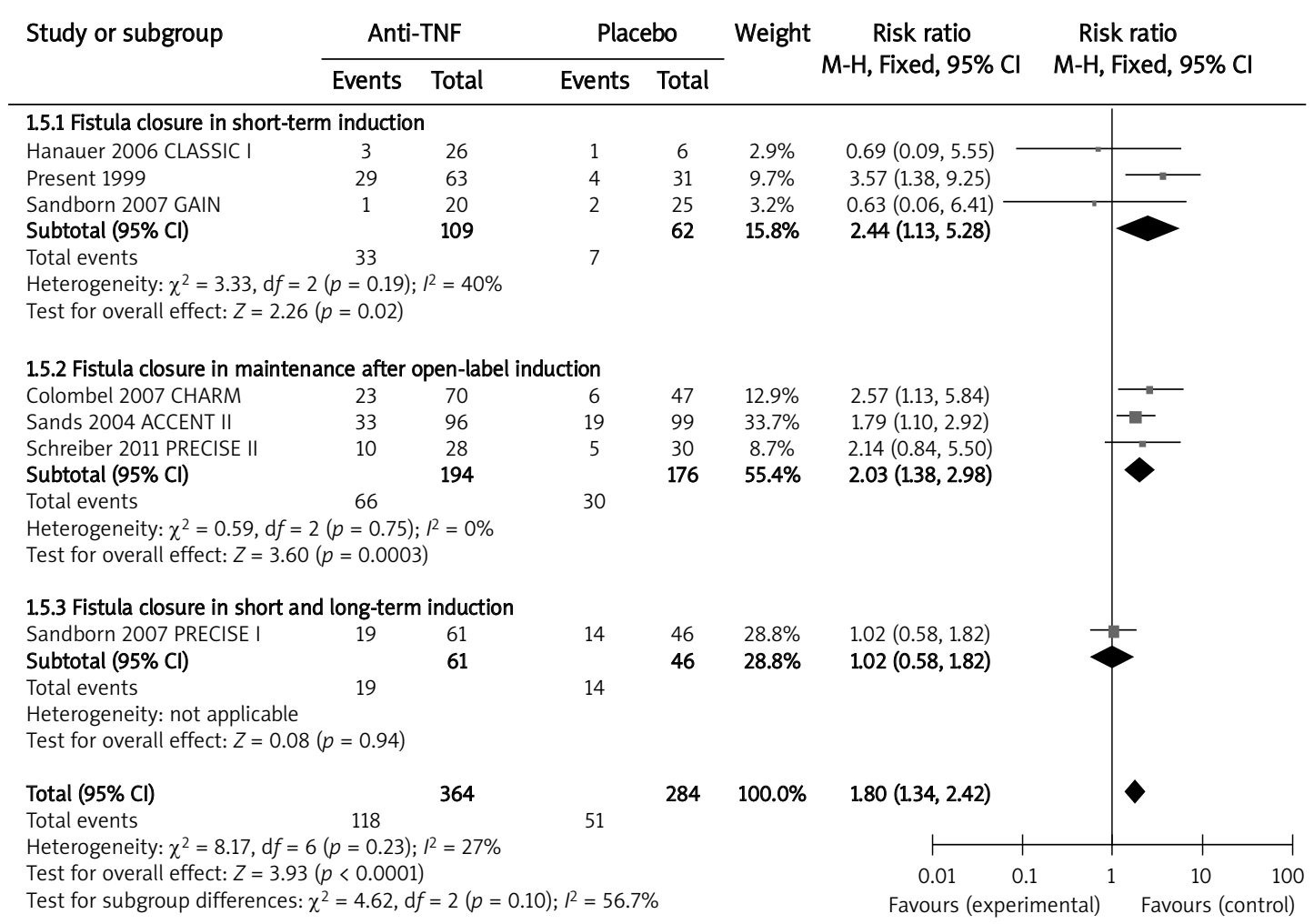

Figure 4. The effect of anti-TNF agents compared with placebo on complete fistula closure 


\begin{tabular}{|c|c|c|c|c|c|c|c|c|c|}
\hline \multirow[t]{2}{*}{ Study or subgroup } & \multicolumn{2}{|c|}{ Anti-TNF } & \multicolumn{2}{|c|}{ Placebo } & \multirow{2}{*}{ Weight } & \multirow{2}{*}{$\begin{array}{c}\text { Risk ratio } \\
\mathrm{M}-\mathrm{H} \text {, Fixed, } 95 \% \mathrm{Cl}\end{array}$} & \multirow{2}{*}{\multicolumn{2}{|c|}{$\begin{array}{c}\text { Risk ratio } \\
\text { M-H, Fixed, 95\% Cl }\end{array}$}} & \\
\hline & Events & Total & Events & Total & & & & & \\
\hline \multicolumn{10}{|l|}{ 1.7.2 Adalimumab } \\
\hline Hanauer 2006 CLASSIC I & 93 & 225 & 19 & 74 & $17.6 \%$ & $1.61(1.06,2.44)$ & & - - & \\
\hline Sandborn 2007 GAIN & 61 & 159 & 41 & 66 & $19.8 \%$ & $0.62(0.47,0.81)$ & - & & \\
\hline Watanabe 2012 & 32 & 67 & 4 & 23 & $10.0 \%$ & $2.75(1.09,6.93)$ & & & \\
\hline Subtotal $(95 \% \mathrm{Cl})$ & & 451 & & 163 & $47.3 \%$ & $1.31(0.53,3.24)$ & & & \\
\hline Total events & 186 & & 64 & & & & & & \\
\hline \multicolumn{10}{|c|}{$\begin{array}{l}\text { Heterogeneity: } \tau^{2}=0.56 ; \chi^{2}=23.04, \mathrm{~d} f=2(p<0.00001) ; I^{2}=91 \% \\
\text { Test for overall effect: } Z=0.58(p=0.56)\end{array}$} \\
\hline \multicolumn{10}{|l|}{ 1.7.3 Certolizumab } \\
\hline Sandborn 2007 PRECISE I & 28 & 331 & 21 & 328 & $15.5 \%$ & $1.32(0.77,2.28)$ & & - & \\
\hline Sandborn 2011 & 76 & 215 & 55 & 209 & $19.5 \%$ & $1.34(1.01,1.80)$ & & - & \\
\hline Winter 2004 & 36 & 65 & 14 & 25 & $17.7 \%$ & $0.99(0.66,1.49)$ & & & \\
\hline Subtotal $(95 \% \mathrm{Cl})$ & & 611 & & 562 & $52.7 \%$ & $123(0.99,1.53)$ & & & \\
\hline Total events & 140 & & 90 & & & & & & \\
\hline \multicolumn{10}{|c|}{$\begin{array}{l}\text { Heterogeneity: } \tau^{2}=0.00 ; \chi^{2}=1.57, \mathrm{~d} f=2(p=0.46) ; 1^{2}=0 \% \\
\text { Test for overall effect: } Z=1.86(p=0.06)\end{array}$} \\
\hline Total $(95 \% \mathrm{Cl})$ & & 1062 & & 725 & $100.0 \%$ & $121(0.81,1.80)$ & & & \\
\hline Total events & 326 & & 154 & & & & & & \\
\hline \multicolumn{10}{|c|}{ Heterogeneity: $\tau^{2}=0.19 ; \chi^{2}=28.18, \mathrm{~d} f=5(p<0.0001) ; 1^{2}=82 \%$} \\
\hline \multirow{2}{*}{\multicolumn{4}{|c|}{$\begin{array}{l}\text { Test for overall effect: } \angle=0.93(p=0.35) \\
\text { Test for subgroup differences: } \chi^{2}=0.02, \mathrm{~d} f=1(p=0.90) ; 1^{2}=0 \%\end{array}$}} & & & 0.01 & & 10 & 100 \\
\hline & & & & & & Favours pla & & Favours ant & \\
\hline
\end{tabular}

Figure 5. The effect of anti-TNF agents compared with placebo on clinical response at week 4; $100-C R$

\begin{tabular}{|c|c|c|c|c|c|c|c|c|c|}
\hline \multirow[t]{2}{*}{ Study or subgroup } & \multicolumn{2}{|c|}{ Anti-TNF } & \multicolumn{2}{|c|}{ Placebo } & \multirow[t]{2}{*}{ Weight } & \multirow{2}{*}{$\begin{array}{c}\text { Risk ratio } \\
\mathrm{M}-\mathrm{H} \text {, Fixed, } 95 \% \mathrm{Cl}\end{array}$} & \multirow{2}{*}{\multicolumn{2}{|c|}{$\begin{array}{c}\text { Risk ratio } \\
\mathrm{M}-\mathrm{H} \text {, Fixed, } 95 \% \mathrm{Cl}\end{array}$}} & \\
\hline & Events & Total & Events & Total & & & & & \\
\hline \multicolumn{10}{|l|}{ 1.9.1 Adalimumab } \\
\hline Colombel 2007 CHARM & 146 & 329 & 28 & 179 & $27.1 \%$ & $2.84(1.98,4.07)$ & & - & \\
\hline Rutgeerts 2012 & 23 & 64 & 9 & 65 & $13.9 \%$ & $2.60(1.30,5.17)$ & & - & \\
\hline Sandborn 2007 CLASSIC II & 31 & 37 & 10 & 18 & $23.3 \%$ & $1.51(0.97,2.33)$ & & - & \\
\hline Subtotal $(95 \% \mathrm{Cl})$ & & 430 & & 262 & $64.3 \%$ & $2.21(1.40,3.51)$ & & & \\
\hline Total events & 200 & & 47 & & & & & & \\
\hline \multicolumn{10}{|c|}{ Heterogeneity: $\tau^{2}=0.10 ; \chi^{2}=5.60, d f=2(p<0.06) ; p^{2}=64 \%$} \\
\hline \multicolumn{10}{|c|}{ Test for overall effect: $Z=3.38(p=0.0007)$} \\
\hline \multicolumn{10}{|l|}{ 1.9.2 Certolizumab } \\
\hline Schreiber 2007 PRECISE ॥ & 135 & 215 & 76 & 210 & $35.7 \%$ & $1.74(1.41,2.13)$ & & 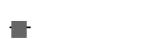 & \\
\hline Subtotal $(95 \% \mathrm{Cl})$ & & 215 & & 210 & $35.7 \%$ & $1.74(1.41,2.13)$ & & & \\
\hline Total events & 135 & & 76 & & & & & & \\
\hline \multicolumn{10}{|l|}{ Heterogeneity: Not applicable } \\
\hline \multicolumn{10}{|c|}{ Test for overall effect: $Z=5.22(p<0.00001)$} \\
\hline Total $(95 \% \mathrm{Cl})$ & & 645 & & 472 & $100.0 \%$ & $2.03(1.48,2.78)$ & & & \\
\hline Total events & 335 & & 123 & & & & & & \\
\hline \multicolumn{10}{|c|}{ Heterogeneity: $\tau^{2}=0.06 ; \chi^{2}=8.09, \mathrm{~d} f=3(p<0.04) ; p^{2}=63 \%$} \\
\hline \multicolumn{6}{|c|}{ Test for overall effect: $Z=4.42(p<0.0001)$} & 0.01 & 1 & 10 & 100 \\
\hline
\end{tabular}

Figure 6. The effect of anti-TNF agents compared with placebo on clinical response during maintenance; $100-C R$ (including study [24])

of the presented results concerned combined data for infliximab and adalimumab but, contrary to study [26], no statistically significant difference was detected between infliximab and adalimumab in the need for dose intensification $(R R=3.28$, 95\% Cl: 0.45-23.95, $p=0.24$ ).

\section{Safety analysis}

We analyzed the incidence of any adverse events (AEs) and serious adverse events (SAEs) both occur- ring as a result of the short-term induction therapy and separately, and incidence of AEs during the long-term maintenance, irrespective of the presence of fistula.

All adverse events or serious adverse events during short-term induction therapy

In the overall analysis, there was no significant difference in the frequency of any AEs between anti-TNF therapy and the placebo $(R R=1.00$, 
95\% Cl: 0.90-1.12, $p=0.97)$ in the short-term induction treatment (range: 4-12 weeks [9, 13-15, 19-22]). In the subgroup analysis, the differences between the anti-TNF agents and placebo were not significant either (infliximab vs. placebo $R R=1.19,95 \%$ $\mathrm{Cl}$ : 0.95-1.49, $p=0.12$; adalimumab vs. placebo RR $=0.88,95 \% \mathrm{Cl}: 0.75-1.03, p=0.11$; certolizumab vs. placebo $\mathrm{RR}=1.06,95 \% \mathrm{Cl}$ : 0.94-1.20, $p=0.32$ ). The results of study [23] were excluded from the abovementioned analysis because the follow-up time was up to 26 weeks, although in the end the inclusion of its results did not affect the overall effect $\left(\mathrm{RR}_{\text {fixed }}=1.00,95 \% \mathrm{Cl}\right.$ : 0.95-1.06, $\left.p=0.91\right)$. The pooled relative risk of the occurrence of SAEs (Figure 7) during the short-term induction treatment (4-12 weeks [15, 19-22], study [23] excluded) indicated the lack of statistical difference between therapies with anti-TNF agents and the placebo $(\mathrm{RR}=1.00,95 \% \mathrm{Cl}: 0.62-1.63, p=0.99)$. There were no data available for infliximab on this outcome. After the inclusion of study [23] the risk was 1.20 (95\% Cl: 0.85-1.71, $p=0.30$ ).

\section{All adverse events or serious adverse events} during long-term maintenance therapy

No statistically significant difference in the incidence of any AEs during the long-term maintenance therapy (48-56 weeks for [10, 12, 16-19], 26 weeks for the PRECISE II study [24]) was detected with the anti-TNF agents when compared with the placebo $(\mathrm{RR}=0.99,95 \% \mathrm{Cl}: 0.93-1.05, p=0.72)$. The overall result of the meta-analysis revealed a statistically significant difference in the frequency of SAEs during the maintenance therapy in favor of the antiTNF agents over the placebo $(\mathrm{RR}=0.70,95 \% \mathrm{Cl}$ :
$0.58-0.86, p=0.0005)[11,12,16-19,24]$, mainly due to a significantly lower risk of SAEs in the subgroup analysis for adalimumab [16-19] (Figure 8). Using the data available from study [26] as a basis, there was no statistically significant difference detected in the risk of any AEs related to the antiTNF therapy with infliximab when compared to adalimumab ( $\mathrm{RR}=0.88,95 \% \mathrm{Cl}$ : 0.69-1.12, $p=0.29$ ).

\section{Discussion}

According to our knowledge, this is the first meta-analysis of the anti-TNF therapy in moderate to severe $C D$, involving fistulizing patients, that includes infliximab, adalimumab and certolizumab. Both infliximab and adalimumab are indicated for moderate to severe $C D$ in patients unresponsive to or intolerant of conventional treatments. Although certolizumab has also shown promising data in adults with $C D$ and received approval from the US FDA, it has not yet been approved by the EMA for that indication.

There were a few limitations for this meta-analysis. First, there are differences in design between the included studies: various periods of treatment with different aims (induction, maintenance), using different doses of agents given at various intervals throughout the studies and irrespective of the concomitant therapy. It is important to note that we performed a meta-analysis for the different types of study design separately: short-term induction trials with randomization before induction, long-term maintenance trials with randomization of responding patients at weeks 2-6 after open-label induction treatment, and one long-term induction trial with randomization before induction. The same method was used for evaluating the effect of the

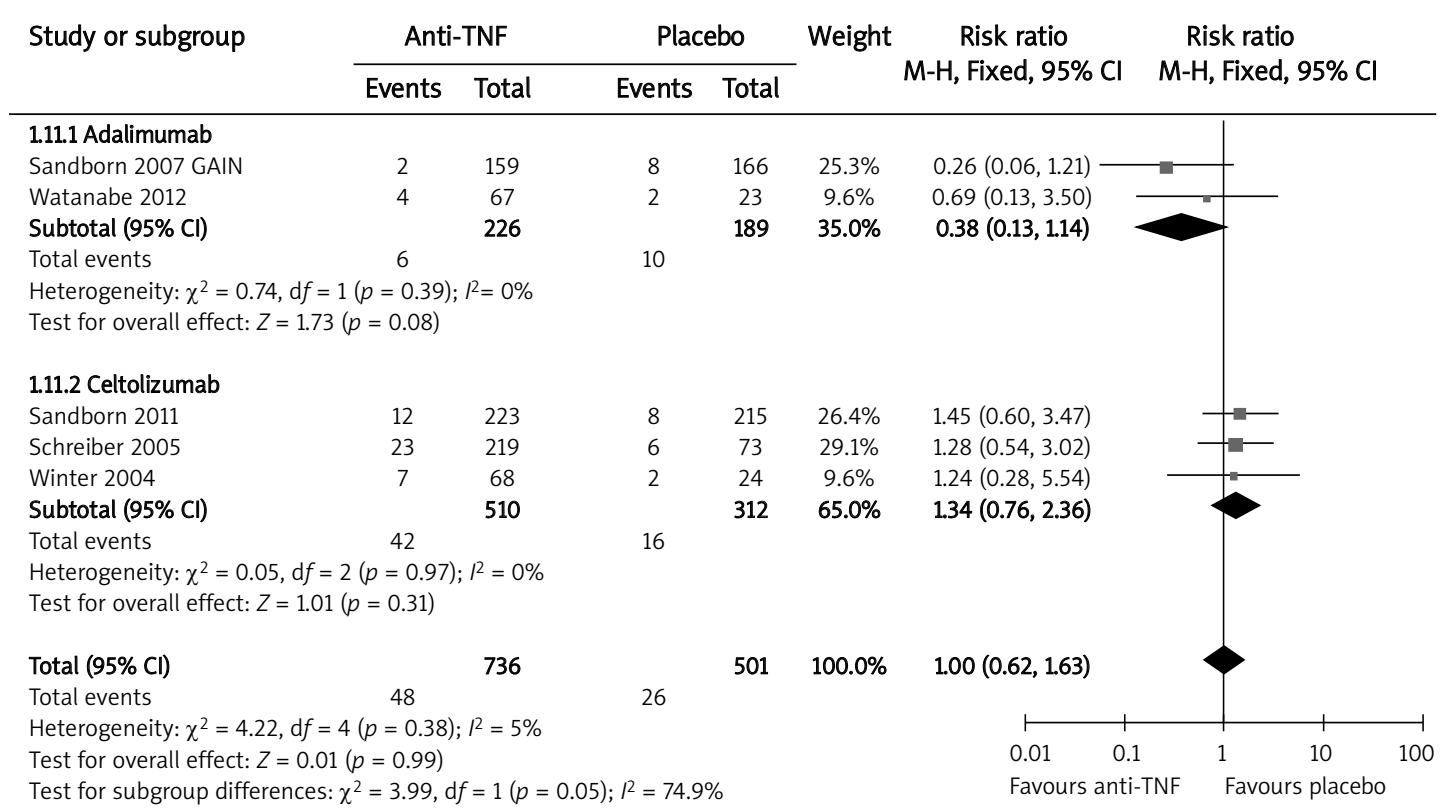

Figure 7. The effect of anti-TNF agents compared with placebo on risk of serious adverse events during induction 


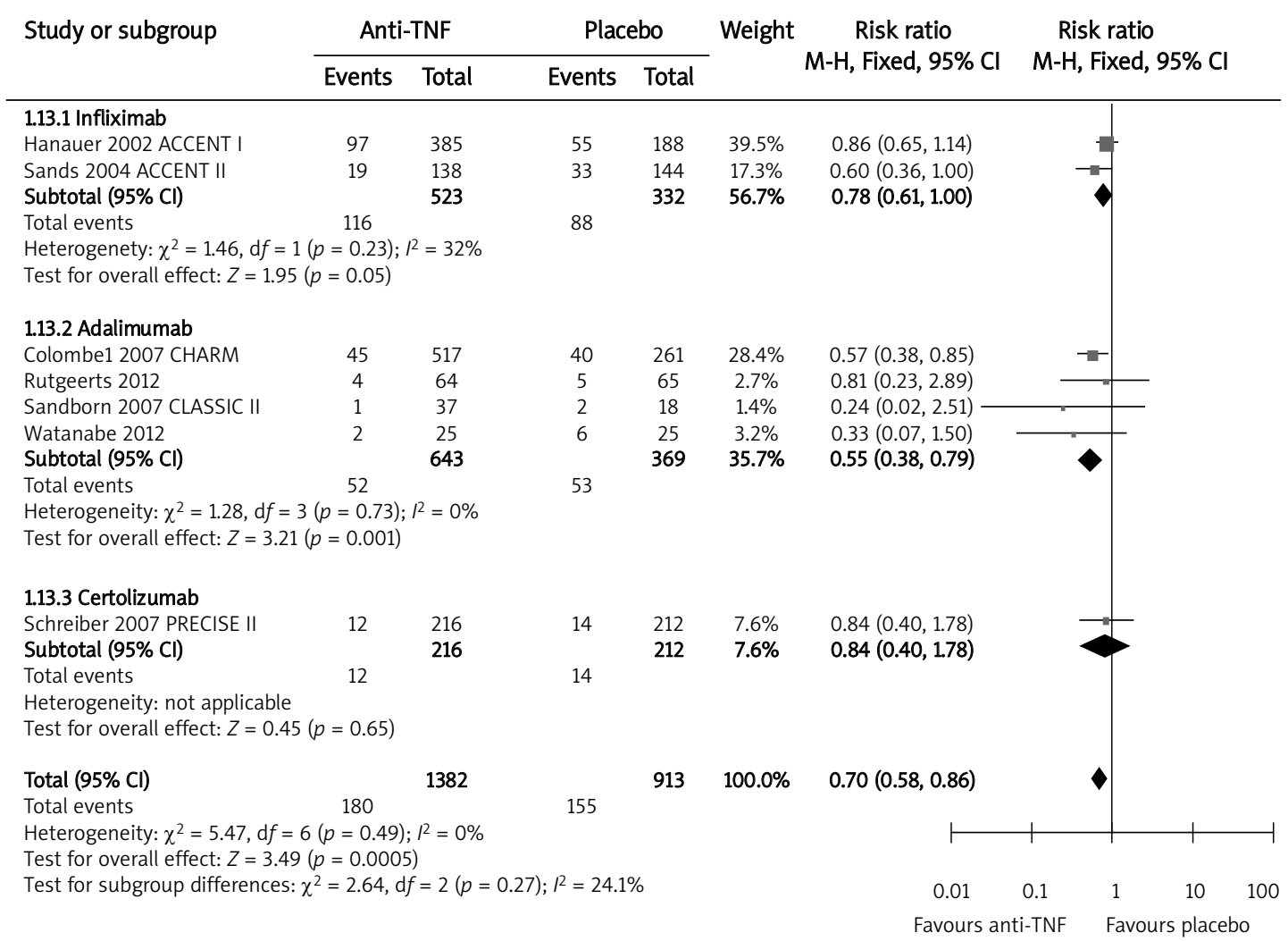

Figure 8. The effect of anti-TNF agents compared with placebo on risk of serious adverse events durning maintenance

anti-TNF agent on fistula healing. Due to the methodological procedure, the limitations, connected with the dissimilar design of the included studies and their duration, did not influence the reliability of the obtained results to a great extent. Both short- and long-term induction studies were used to evaluate clinical remission and response rates at week 4 (for all the induction trials these data were extractable, although some studies lasted up to 12 weeks). For the long-term maintenance studies, the follow-up periods varied between 48 and 56 weeks, with one study lasting only 26 weeks [24], and the last point of the trial follow-up was used to extract the outcomes of the data wherever trial reporting allowed it (likewise for the safety analysis in both the induction and maintenance therapy). In this setting, we carried out an additional analysis based on extractable data on the maintenance of remission at 20-30 weeks, with regard to the study PRECISE II [24]. Apart from pooling the results from studies of different anti-TNF agents, different doses and slightly different durations of therapy (except for the clinical outcomes at week 4), we performed subgroup analyses for each of the anti-TNF agents in order to investigate potential differences in their efficacy. The results of the maintenance trials were almost exclusively based on the subgroups of the 'responders', which were select- ed for the analysis or randomization at varying time points after an induction period, during which all the patients received the study drug. Separate results for non-responders are not reported here. What should be noted, including study [18] for the maintenance of remission after open-label induction could bias the overall results, because for this efficacy endpoint we included studies evaluating the maintenance of remission among responders after open-label induction, while maintenance treatment in study [18] was conducted among patients probably not strictly preselected for a response to induction treatment. Additionally, the meta-analysis included the GAIN study [15], where adalimumab was given to patients who did not achieve a response to previous infliximab therapy, thus possibly leading to an underestimation of the real efficacy of the anti-TNF agents in this setting. In regards to evaluation of the treatment effects on fistula healing, except data from two trials designed specifically to address this issue $[12,13]$, we extracted welldefined data on fistula closure from other primary studies also including patients with fistulizing disease, but it should be noted that patients included in the studies $[12,13]$ have more severe CD compared to all other studies included in this comparison, in which patients had moderately to severely active CD, defined as a CDAl score between 220 and 
450. What is important, individual results from studies designed with healing of fistulas as the primary outcome of interest demonstrated a clear, statistically significant benefit of infliximab as well as adalimumab over placebo, even in more severe disease $[12,13]$. Nevertheless, even after taking into consideration the results of this particular study there should be no difference in the reliability and statistical significance of the final results of the metaanalysis.

We identified two studies regarding a head-tohead comparison between infliximab and adalimumab $[26,27]$ in treatment for $C D$, but taking into consideration the differences in their methodology and quality, pooling the results was not recommended and a narrative synthesis was performed instead.

Several meta-analyses have shown the efficacy of anti-TNF therapy for inducing and maintaining clinical response and remission in patients with CD. It is a fact that these meta-analyses and reviews were performed with less contemporaneous research data and did not therefore consider the most recent studies published in 2011 and 2012. Peyrin-Biroulet et al. [28] performed a meta-analysis comparing the effectiveness of six anti-TNF agents (infliximab, adalimumab, certolizumab, etanercept, onercept, and CDP571) with a placebo for induction and maintenance, steroid-sparing and fistula closure in CD. The methods of conducting that analysis were similar to ours. Based on 21 trials of which 16 were included in the efficacy analysis, there was evidence that the anti-TNF therapy is effective in patients with $C D$ refractory to standard medical therapy, but the authors stated that the efficacy of the anti-TNF agents besides infliximab in the treatment of fistulizing patients requires additional investigation. Safety analyses revealed that the antiTNF therapy does not increase the risk of death, serious infection or malignancy. However, subgroup analyses were performed, and the results of the overall meta-analysis also contain particular data from studies evaluating an anti-TNF agent other than antibodies (etanercept or onercept). The results of review [29] showed the superiority of infliximab, adalimumab and certolizumab (separately) in relation to the placebo for the maintenance of remission in patients who have responded to prior induction therapy, but only infliximab was effective for the maintenance of fistula healing. The review also has two drawbacks. Firstly, it encompasses a small number of studies: three concerning infliximab, then two and one examining adalimumab and certolizumab, respectively. Secondly, the pooled results consisted of data from no more than two studies. This was caused by the fact that no overall (all anti-TNF agents combined) metaanalysis was performed. Results from 11 trials included in a more recent systematic review [30] showed that both infliximab and adalimumab were beneficial for induction and maintenance therapy in adults with moderate to severe $C D$, and infliximab only for adults with a fistula when compared to a placebo. Certolizumab was not assessed. It also needs to be highlighted that the data from studies included in review [30] were not pooled in the metaanalysis, and, for that reason, the conclusion from that descriptive analysis should be interpreted cautiously. In review [31] there is an assessment of biological therapies (three anti-TNF antibodies and anti-integrin antibody) for Crohn's disease and ulcerative colitis (UC) treatment. In the overall analysis, the anti-TNF agents (certolizumab, adalimumab, and infliximab combined) were significantly more effective than the placebo for inducing remission and preventing relapse of $C D$, although the pooled data from six studies demonstrated no statistically significant difference in regard to healing of fistulizing CD. In review [31], as described in the data extraction methodology, the data concerning the outcomes were extracted at the last point of the trial follow-up. It means that, as opposed to our analysis, the data for the induction of remission were extracted at different time points due to the fact that the eligible time of outcome assessment for induction trials was up to 4 months (note that induction therapy in study [23] lasted 26 weeks). In addition, the combined result for fistula healing was presented without explanation of its definition (complete or partial closure). Our results on induction of remission or clinical response at week 4 are contrary to results obtained in the metaanalysis by Rahimi et al., 2011 [32]. That meta-analysis included studies in which patients with CD were exposed to any therapeutic dosage of any anti-TNF agents (infliximab, certolizumab, CDP870, CDP571, etanercept, onercept) and shared some trials included in the current analysis. The results demonstrated that anti-TNF agents have no statistically significant effects on induction of remission after 2 weeks of treatment as well as clinical response and remission after 4 weeks. The only statistically significant benefit of anti-TNF agents over the placebo was observed for improving clinical response after 2 weeks of treatment. What is important, relevant data included in meta-analysis [32] were collected up to December 2005. On the other hand, results from a meta-analysis including studies concerning severe ulcerative colitis [33] showed that infliximab was significantly more beneficial than placebo for induction of remission at 2-8 weeks and slightly, but not significantly better than corticosteroids in regards to the induction of clinical remission up to week 13.

Fistulas, one of the most severe complications of $C D$, causes impairment in the quality of life and 
increase the likelihood of total colectomy. Perianal fistula is the most common fistula type, occurring in $25-50 \%$ of patients with CD $[12,25]$. As mentioned above, some meta-analyses of anti-TNF antibodies have shown success with their use in fistulizing $C D$, but it particularly concerns infliximab [28-30]. Apart from surgery, another method of fistula treatment is short-term therapy with antibiotics. In a review [34] pooling of data showed that immunoregulators and antibiotics are significantly more effective than placebo for $\geq 50 \%$ as well as complete fistula closure in fistulizing $C D$. In recent years treatment with anti-TNF antibodies has significantly improved the management of CD as well as UC refractory to conventional therapies; nevertheless, there is still a large number of patients showing an evaluative and disabling course of disease who require surgery [3]. Pre-operative use of biological therapy such as anti-TNF agents may potentially increase the risk for postoperative infectious complications by suppression of the immune response. In the meta-analysis performed by Ehteshami-Afshar et al., 2011 [2], there was no evidence of significant differences in the rate of colectomy, post-colectomy infectious complications or deaths in patients with ulcerative colitis who received infliximab in comparison to those who did not. After pooling results from studies assessing $U C$ and $C D$, the overall early post-operative complications were more frequent among patients who received infliximab pre-operatively (but not late post-operative complications). Nonetheless, the rate of post-operative hospitalization was significantly lower in patients with UC under infliximab therapy [2].

Overall, anti-TNF therapy is a relatively safe treatment option in CD and other inflammatory bowel diseases [1]. Results of our meta-analyses demonstrated no significant differences in the frequency of any adverse events, including serious AEs, between anti-TNF therapy and placebo during shortterm treatment. Moreover, there was a significantly lower risk of SAEs during maintenance therapy in favor of anti-TNF agents over placebo.

The overall results of our meta-analysis indicated consistent superiority of the anti-TNF antibodies when compared to placebo in the induction and maintenance of clinical remission, but notably, in contrast to reviews [28,31], we demonstrated their significant beneficial effect on fistula healing also in short-term induction therapy. What should be emphasized is that we took into consideration such outcomes as clinical response (CR-70, CR-100), which was evaluated separately for both short-term induction and maintenance therapy, and has not been meta-analyzed in any previous reviews, except for article [29], where the attention was only on maintenance therapy in patients who had already had remission after induction therapy, and article
[32] concerning the short-term induction phase. In contrast to previous articles, our updated review mentions the newest studies evaluating two of the anti-TNF antibodies in comparison with each other.

With respect to previous review studies, our systematic review is the most up-to-date systematic review (including studies published in 2012) on the effect of anti-TNF antibodies in CD, which in its methodology followed the methods described in the Cochrane Handbook, with the report having been prepared according to the PRISMA statement.

In conclusion, in general, the results of the metaanalysis demonstrated that anti-TNF therapy has a beneficial effect on induction and maintenance of clinical remission and response, as well as fistula healing, when compared with placebo. Based on a safety analysis, there was no evidence for an increase in the incidence of any adverse events related to anti-TNF when compared with placebo. In conclusion, the risk-benefit ratio of therapy with infliximab, adalimumab or certolizumab in CD is therefore in favor of these agents.

\section{References}

1. Magro F, Pórtela F. Management of inflammatory bowel disease with infliximab and other anti-tumor necrosis factor alpha therapies. Biodrugs 2010; 24 Suppl. 1: 3-14.

2. Ehteshami-Afshar S, Nikfar S, Rezaie A, Abdollahi M. A systematic review and meta-analysis of the effects of infliximab on the rate of colectomy and post-operative complications in patients with inflammatory bowel disease. Arch Med Sci 2011; 7: 1000-12.

3. Spurio FF, Aratari A, Margagnoni G, Doddato MT, Papi C. Early treatment in Crohn's disease: do we have enough evidence to reverse the therapeutic pyramid? J Gastrointestin Liver Dis 2012; 21: 67-73.

4. Higgins JPT, Green S. Cochrane handbook for systematic reviews of interventions Version 5.1.0 [updated March 2011]. Cochrane Collaboration and John Wiley, 2011. Available from: www.cochrane-handbook.org

5. Moher D, Liberati A, Tetzlaff J, et al. The PRISMA Group (2009). Preferred Reporting Items for Systematic Reviews and Meta-Analyses: The PRISMA Statement. PLoS Med 2009; 6: e1000097.

6. Jadad AR, Moore RA, Carroll D, et al. Assessing the quality of reports of randomized clinical trials: is blinding necessary? Control Clin Trials 1996; 17: 1-12.

7. Wells GA, Shea B, O'Connell D, et al. The NewcastleOttawa Scale (NOS) for assessing the quality of nonrandomised studies in meta-analyses. Available from: URL: http://www.ohri.ca/programs/clinical_epidemiology/ oxford.asp

8. Su C, Lichtenstein GR, Krok K, et al. A meta-analysis of the placebo rates of remission and response in clinical trials of active Crohn's disease. Gastroenterology 2004; 126: 1257-69.

9. Targan S, Hanauer SB, van Deventer SJH, et al. A short term study of chimeric monoclonal antibody CA2 to tumour necrosis factor alpha for Crohn's disease. N Engl J Med 1997; 337: 1029-35.

10. Rutgeerts P, D'Haens G, Targan S, et al. Efficacy and safety of retreatment with anti-tumor necrosis factor antibody 
(infliximab) to maintain remission in Crohn's disease. Gastroenterology 1999; 117: 761-9.

11. Hanauer SB, Feagan BG, Lichtenstein GR, et al. Maintenance infliximab for Crohn's disease: the ACCENT I randomised trial. Lancet 2002; 359: 1541-9.

12. Sands BE, Anderson FH, Bernstein CN, et al. Infliximab maintenance therapy for fistulizing Crohn's disease. N Engl J Med 2004; 350: 876-85.

13. Present $D H$, Rutgeerts $P$, Targan $S$, et al. Infliximab for the treatment of fistulae in patients with Crohn's disease. N Engl J Med 1999; 340: 1398-405.

14. Hanauer SB, Sandborn WJ, Rutgeerts P, et al. Human antitumor necrosis factor monoclonal antibody (adalimumab) in Crohn's disease: The CLASSIC-I trial. Gastroenterology 2006; 130: 323-32.

15. Sandborn WJ, Rutgeerts P, Enns R, et al. Adalimumab induction therapy for Crohn disease previously treated with infliximab: a randomized trial. Ann Intern Med 2007; 146: 829-38.

16. Sandborn WJ, Hanauer SB, Rutgeerts PJ, et al. Adalimumab for maintenance treatment of Crohn's disease: results of the CLASSIC II Trial. Gut 2007; 56: 1232-9.

17. Colombel J, Sandborn WJ, Rutgeerts P, et al. Adalimumab for maintenance of clinical response and remission in patients with Crohn's disease: The CHARM Trial. Gastroenterology 2007; 132: 52-65.

18. Rutgeerts P, Van Assche G, Sandborn WJ, et al. Adalimumab induces and maintains mucosal healing in patients with Crohn's disease: data from the EXTEND trial. Gastroenterology 2012; 142: 1102-11.

19. Watanabe M, Hibi T, Lomax KG, et al. Adalimumab for the induction and maintenance of clinical remission in Japanese patients with Crohn's disease. J Crohns Colitis 2012; 6: 160-73.

20. Winter TA, Wright J, Ghosh S, Jahnsen J, Innes A, Round P. Intravenous CDP870, a PEGylated Fab' fragment of a humanized antitumour necrosis factor antibody, in patients with moderate-to-severe Crohn's disease: an exploratory study. Aliment Pharmacol Ther 2004; 20 : 1337-46.

21. Schreiber S, Rutgeerts P, Fedorak RN, et al. A randomized, placebo-controlled trial of certolizumab pegol (CDP870) for treatment of Crohn's disease. Gastroenterology 2005; 129: 807-18.

22. Sandborn WJ, Schreiber S, Feagan BG, et al. Certolizumab pegol for active Crohn's disease: a placebo-controlled, randomized trial. Clin Gastroenterol Hepatol 2011; 9: 670-8.

23. Sandborn WJ, Feagan BG, Sloinov S, et al. Certolizumab pegol for the treatment of Crohn's disease. N Engl J Med 2007; 357: 228-38

24. Schreiber S, Khaliq-Kareemi M, Lawrance IC, et al. Maintenance therapy with certolizumab pegol for Crohn's disease. N Engl J Med 2007; 357: 239-50.

25. Schreiber S, Lawrance IC, Thomsen OØ, Hanauer SB, Bloomfield R, Sandborn WJ. Randomised clinical trial: certolizumab pegol for fistulas in Crohn's disease subgroup results from a placebo-controlled study. Aliment Pharmacol Ther 2011; 33: 185-93.

26. Van Assche G, Vermeire S, Ballet V, et al. Switch to adalimumab in patients with Crohn's disease controlled by maintenance infliximab: prospective randomised SWITCH trial. Gut 2012; 61: 229-34.

27. Riis A, Martinsen TC, Waldum HL, Fossmark R. Clinical experience with infliximab and adalimumab in a singlecenter cohort of patients with Crohn's disease. Scand J Gastroenterol 2012; 47: 649-57.
28. Peyrin-Biroulet L, Deltenre P, de Suray N, Branche J, Sandborn WJ, Colombel JF. Efficacy and safety of tumor necrosis factor antagonists in Crohn's disease: metaanalysis of placebo-controlled trials. Clin Gastroenterol Hepatol 2008; 6: 644-53.

29. Behm BW, Bickston SJ. Tumor necrosis factor-alpha antibody for maintenance of remission in Crohn's disease. Cochrane Database Syst Rev 2008; 23: CD006893.

30. Dretzke J, Edlin R, Round J, et al. A systematic review and economic evaluation of the use of tumour necrosis factoralpha (TNF-alpha) inhibitors, adalimumab and infliximab, for Crohn's disease. Health Technol Assess 2011; 15: 1-244.

31. Ford AC, Sandborn WJ, Khan KJ, Hanauer SB, Talley NJ, Moayyedi P. Efficacy of biological therapies in inflammatory bowel disease: systematic review and meta-analysis. Am J Gastroenterol 2011; 106: 644-59.

32. Rahimi R, Nikfar S, Abdollahi M. Do anti-tumor necrosis factors induce response and remission in patients with acute refractory Crohn's disease? A systematic metaanalysis of controlled clinical trials. Biomed Pharmacother 2007; 61: 75-80

33. Nikfar S, Ehteshami-Afshar S, Abdollahi M. A systematic review and meta-analysis of the efficacy and adverse events of infliximab in comparison to corticosteroids and placebo in active ulcerative colitis. Intern J Pharmacol 2011; 7: 325-32.

34. Nikfar S, Mirfazaelian H, Abdollahi M. Efficacy and tolerability of immunoregulators and antibiotics in fistulizing Crohn's disease: a systematic review and meta-analysis of placebo-controlled trials. Curr Pharm Des 2010; 16: 3684-98. 